A C G

publications

Org. Commun. 14:1 (2021) 73-80

organic

communications

\title{
Swelling and durability performance of surface-grafted polymer brushes and brush gels
}

\author{
Selin Kinali-Demirci ${ }^{1,2}$ and Serkan Demirci ${ }^{1,2 *}$ \\ ${ }^{1}$ Department of Chemistry, Amasya University, Ipekkoy 05100 Amasya, Türkiye \\ ${ }^{2}$ Department of Biotechnology, Amasya University, Ipekkoy 05100 Amasya, Türkiye
}

(Received December 07, 2020; Revised March 03, 2021; Accepted March 05, 2021)

\begin{abstract}
Polymer brushes and brush gels were prepared by RAFT polymerization. Prepared surfaces were characterized by XPS, AFM, ellipsometry, and water contact angle measurements. The swelling properties and stability of surfaces were compared. It was determined that the swelling ratio decreased with increasing grafting density and cross-linker ratio for polymer brushes and brush gels, respectively. As a result, it was observed that crosslinked polymer brushes were more stable than polymer brushes, and the stability and swelling properties of polymer brushes could be controlled by cross-linking.
\end{abstract}

Keywords: Polymer brush; brush gels; polymeric ionic liquid; RAFT polymerization. (C2021 ACG Publication. All right reserved.

\section{Introduction}

In 1914, Paul Walden first described ionic liquids (ILs) in his publication, where he gave the physical properties of ethyl ammonium nitrate. ${ }^{1}$ Even though in the years it was discovered it did not receive adequate attention, the exploration of this new generation material is considered to be around 100 years old, and today they have managed to become the center of attention with ion conductivity, high thermal stability, non-flammability, high heat capacity, low vapor pressure, high polarity, and dissolving power. ${ }^{2,3}$ However, in the industrial processing of chemicals, it has often been labeled "green" and an environmentally friendly solvent. ${ }^{4}$

Polymeric ionic liquids obtained by polymerization of ionic liquids are a subgroup of polyelectrolytes. ${ }^{5}$ This new generation polymeric materials have been obtained by incorporating ionic liquids' unique properties into the polymer chain. The polymeric materials in this subject have attracted attention in many fields of science and technology, from surface applications to temperature-sensitive and porous materials, from energy to biotechnology. ${ }^{6-8}$

Thin organic coatings are often used to control the interaction of the coated material with the environment. Functional coatings have found their way in different high-tech applications such as electronics and biotechnology. ${ }^{9,10}$ Two different methods are used to prepare organic coatings depending on the interaction between the molecules and substrate. A common feature of physical methods is the dissolution of molecules in suitable solvents and then evaporation of the solvent. ${ }^{11}$ However, chemical methods in which molecules are covalently attached on solid surfaces (for example, polymer brushes) are more stable than physical methods. ${ }^{12-14}$ Although various polymerization techniques can be used for polymer brush synthesis, reversible-deactivation radical polymerization techniques are used in many studies due to their advantages. These techniques allow the preparation of well-defined polymers with

\footnotetext{
*Corresponding author:E-Mail: srkndemirci@gmail.com
} 
controlled molecular weight, polydispersity, composition, chain architecture, and site-specific functionality. ${ }^{15-19}$

Although, compared to physical methods, chemical methods put forth more stable coatings, ${ }^{20}$ depending on the conditions in which they are used, all thin organic films remove from the surface over time. This condition presents important problems, especially for polymer brushes bonded to the surface from a single point. To address these problems, cross-linked polymer brushes (brush gels) have been developed. ${ }^{21-23}$ Unlike polymer brushes attached to a solid surface by covalent bonds, brush-gels contain cross-linker and offer a combination of both polymer brushes and gels. In this study, the swelling behavior and stability of polymer brushes and brush gels were investigated. Consequently, the brush gels are more stable, although they showed similar swelling behavior with polymer brushes.

\section{Experimental}

\subsection{Materials and Methods}

All chemicals were analytical reagent grade and obtained from Sigma-Aldrich and used as purchased unless otherwise specified. Deionized water (18.2 M $2 . \mathrm{cm})$ was obtained from a Milli-Q water purification system (Millipore, Bedford, MA, USA). The chemical composition information of the samples was obtained by XPS; the measurement was carried out on a Thermo Scientific K-Alpha spectrometer using a monochromatic Al K- $\alpha$ X-ray source $(h v=1486.6 \mathrm{eV})$. Charging neutralizing equipment was used to compensate sample charging, and the binding scale was referenced to the aliphatic component of $\mathrm{C} 1 \mathrm{~s}$ spectra at $285.0 \mathrm{eV}$. The water contact angle measurements were conducted at room temperature using a goniometer (DSA 100, Krüss) equipped with a microliter syringe. Deionized water $(5.0 \mu \mathrm{L})$ was used as the wetting liquid. The morphology of the silicon wafers was recorded on a Park Systems XE70 SPM Controller LSF-100 HS. A triangular-shaped $\mathrm{Si}_{3} \mathrm{~N}_{4}$ cantilever with integrated tips (Olympus) was used to acquire the images in the non-contact mode. The normal spring constant of the cantilever was $0.02 \mathrm{~N} / \mathrm{m}$. The force between the tip and the sample was $0.87 \mathrm{nN}$. The ellipsometric measurements were performed under ambient conditions using an ellipsometer (model DRE, EL X20C) equipped with a He-Ne laser $(\lambda=632.8 \mathrm{~nm})$ at a constant incident angle of $75^{\circ}$. The average dry thickness of polymer brushes on silicon substrate was determined by fitting the data with a three-layer model (native silicon [refractive index, $n=3.86]+$ silicon oxide layer $[n=1.46]+$ organic layer [ $n=1.47]$. The swelling ratio of Ba-c and CBa-c layers were determined with ellipsometric thickness. The dry ellipsometric thickness $\left(h_{d}\right)$ of polymer-coated layers was measured, then the Ba-c and CBa-c layers were immersed in deionized water until equilibrium was reached. After the swollen Ba-c and CBa-c layers were removed from the aqueous solution, and wet thickness $\left(h_{s}\right)$ of the Ba-c and CBa-c layers was measured. To examine the durability of Ba-c and CBa-c layers, a homemade rotary flow system was used. ${ }^{20} \mathbf{B a}-\mathbf{c}$ and $\mathbf{C B a}-\mathbf{c}$ grafted silicon wafers were placed in a silicon tube (inner diameter: $10 \mathrm{~mm}$, length: $310 \mathrm{~mm}$ ) with PBS solution $(10 \mathrm{~mL})$. The homemade rotary flow system was connected to the evaporator (Heidolph Hei-VAP), was rotated at $37{ }^{\circ} \mathrm{C}$ for $72 \mathrm{~h}$. The substrates were washed with PBS and water dried under a stream of nitrogen.

\subsection{Synthesis of 1-vinyl-3-buthylimidazolium bromide (VBIMBr)}

A mixture containing 1-vinylimidazole $(10.00 \mathrm{~g}, 0.11 \mathrm{~mol})$ and equivalent molar amount of 1bromobutane $(14.56 \mathrm{~g}, 0.11 \mathrm{~mol})$ was stirred at room temperature for $48 \mathrm{~h}$. The product was washed three times with diethyl ether and ethyl acetate and then dried in vacuum at room temperature to yield 1-vinyl-3-buthylimidazolium bromide (88\% yield). Physical and spectroscopic data were in agreement with literature reports. ${ }^{24}$

\subsection{Synthesis of 1,4-di(3-vinylimidazolium)butane bisbromide (DVIMBr)}

1,4-Di(3-vinylimidazolium)butane bisbromide (DVIMBr) was prepared by standard method. ${ }^{25}$ 1-Vinylimidazole ( $1 \mathrm{~g}, 10.6 \mathrm{mmol})$ and 1,4-dibromobutane (1.15 g, $5.3 \mathrm{mmol})$ were stirred in methanol at $60{ }^{\circ} \mathrm{C}$ for $15 \mathrm{~h}$. Upon cooling, the reaction mixture was poured dropwise into diethyl ether. The white precipitate was filtered off and dried at room temperature (61\% yield). Physical and spectroscopic data were in agreement with literature reports. ${ }^{25}$ 


\subsubsection{Surface Fabrication}

RAFT agent immobilized silicon wafers, which were prepared in our previous reports, ${ }^{26-28}$ were placed in a glass reactor and degassed under vacuum for $30 \mathrm{~min}$. VBIMBr monomer and DVIMBr crosslinker (1, 2, and 5\% of monomer for CBa-c) were added to a dry Schlenk tube along with 4-cyano-4(phenylcarbonothioylthio)pentanoic acid (CPAD), 4,4'-azobis(4-cyanovaleric acid) (ACVA) (Monomer/RAFT agent/Initiator: 200:2:1), and DMF (10 mL). The mixture was degassed by three freeze-pump-thaw cycles. The solution was transferred to the glass reactor just before heating the reactor to $70{ }^{\circ} \mathrm{C}$ for $24 \mathrm{~h}$ to allow for polymerization, and cross-linked PILs were obtained. The modified silicon wafers were washed with acetone and dried under a stream of nitrogen.

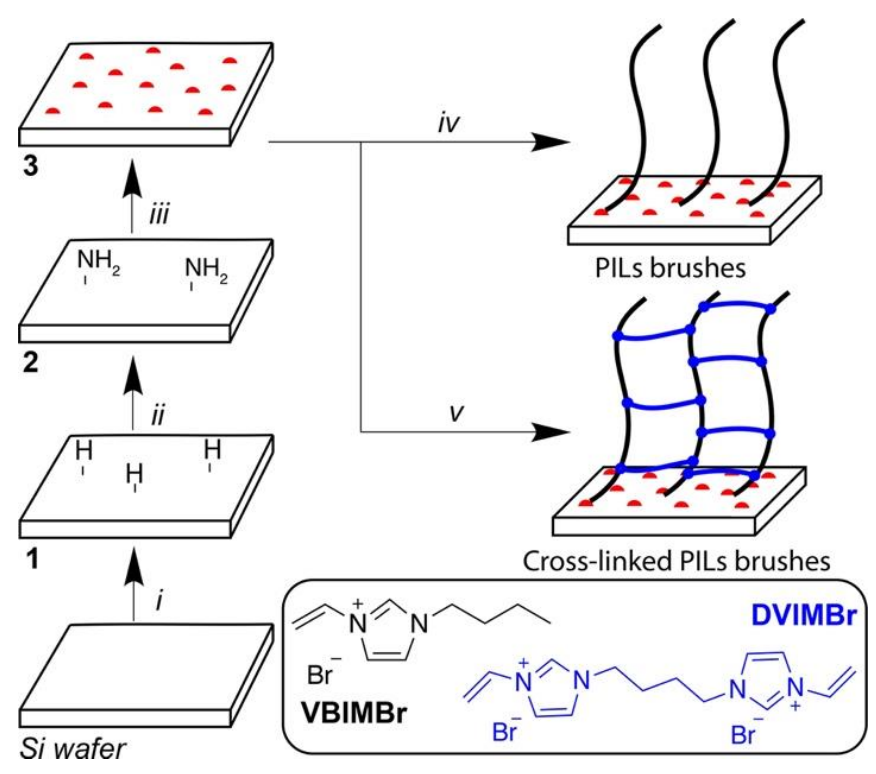

Scheme 1. Preparation of PIL brushes (Ba-c) and brush gels (CBa-c). $i$. Piranha solution, 2\% HF; ii. $t$-BOC allylamine - UV, 25\% TFA, $10 \% \mathrm{NH}_{4} \mathrm{OH}$; iii. CPDBE, DCM, $60 \mathrm{~h}$, rt; $i v$. VBIMBr, CPAD, ACVA, $24 \mathrm{~h} 70{ }^{\circ} \mathrm{C} ; v$. VBIMBr, DVIMBr, CPAD, ACVA, $24 \mathrm{~h} 70{ }^{\circ} \mathrm{C}$. Chemical structures of VBIMBr and DVIMBr

\section{Results and Discussion}

\subsection{Surface Modification and Characterization}

Polymer brushes and brush gels were prepared by RAFT polymerization. Silicon surfaces were cleaned with HF (1), and prepared hydrogen-terminated surfaces were modified with allylamine using UV (2). RAFT agent immobilized on amine-terminated surfaces, and polymer brushes were synthesized via RAFT polymerization (Scheme 1). The cross-linked polymer brushes were synthesized via in situ cross-linking method with different cross-linker ratios.

Table 1. Atomic concentration of PIL layers as determined by XPS analysis

\begin{tabular}{lcccc}
\hline \multirow{2}{*}{ Entry } & \multicolumn{4}{c}{ Atomic concentration (\%) } \\
\cline { 2 - 5 } & $\mathbf{C}$ & $\mathbf{N}$ & $\mathbf{O}$ & $\mathbf{B r}$ \\
\hline Ba & 68.13 & 15.19 & 9.21 & 7.47 \\
Bb & 67.51 & 14.76 & 10.45 & 7.28 \\
Bc & 66.73 & 14.51 & 11.79 & 6.97 \\
CBa & 66.27 & 15.56 & 10.34 & 7.83 \\
CBb & 66.31 & 15.67 & 9.77 & 8.25 \\
CBc & 64.52 & 16.24 & 10.35 & 8.89 \\
\hline
\end{tabular}



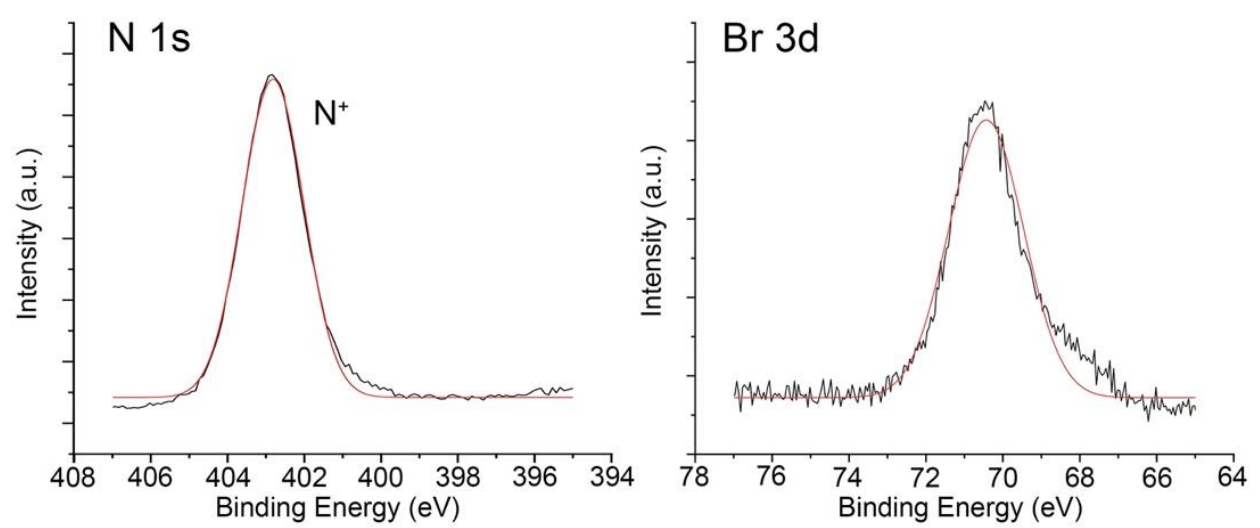

Figure 1. High resolution N 1s and Br 3d XPS spectrum of PILs brushes. The black solid curves represent the measured spectra, and the red solid curves represent the fitted results

The synthesis and characterization of surfaces modified with a RAFT agent (3) have been detailed in our previous studies. ${ }^{26-28}$ After RAFT polymerization, polymer brushes and brush gels were determined by the $\mathrm{N} 1 \mathrm{~s}$ signal caused by positively charged nitrogen $(402.3 \mathrm{eV})$ and $\mathrm{Br} 3 \mathrm{~d}(70.4 \mathrm{eV})$ (Figure 1). The chemical composition of the prepared surfaces was summarized in Table 1. It has been observed that the N/C ratio increased with the increase of the cross-linker ratio. However, the N/C ratios were lower than the theoretical N/C ratios for all cross-linked polymer brushes. This indicates that the cross-linker ratios of brush gels were lower than initial feed ratios.

The morphologies of prepared surfaces were determined by atomic force microscopy (AFM). Two-and three-dimensional AFM images are shown in Figure 2. It was determined that the non-crosslinked surfaces (Ba-c) were needle-like structure and that the surface roughness was $3.457 \mathrm{~nm}$. RMS values increased from $3.682 \mathrm{~nm}$ to $7.831 \mathrm{~nm}$, with an increased cross-link ratio. The AFM images showed that, the needle-like structure turned into a rod-like structure with cross-linking.
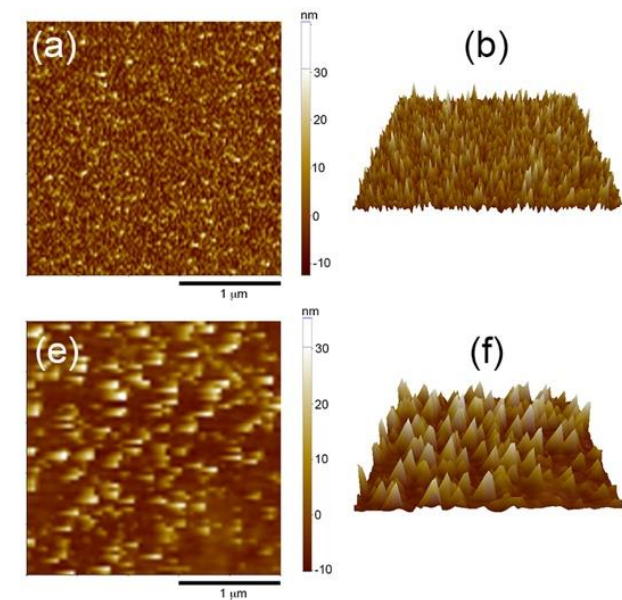

(f)

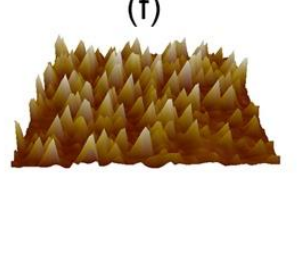

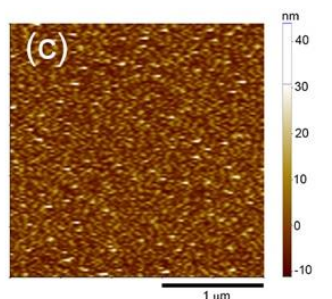

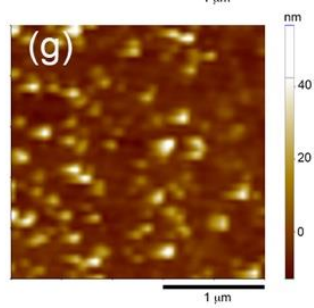

(d)

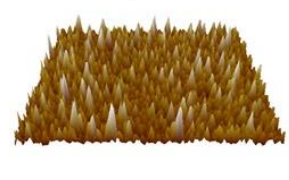

(h)

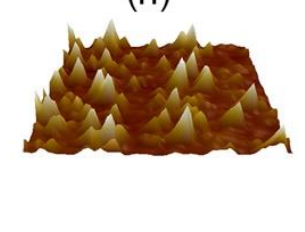

Figure 2. 2D and 3D surface topography of $2.5 \mu \mathrm{m}$ images of $\mathbf{B a}(\mathrm{a}, \mathrm{b}), \mathbf{C B a}(\mathrm{c}, \mathrm{d}), \mathbf{C B b}(\mathrm{e}, \mathrm{f})$ and $\mathbf{C B c}$ $(\mathrm{g}, \mathrm{h})$

The grafting density $(\sigma)$ and average distance between grafting points, $D$ of the polymer brushes were calculated from the dry polymer thickness, $h$ and the number-average molar weight, $M_{n}$ (the molecular weight of the grafted polymer chains is assumed to be similar to that of the free polymer in solution) values using eqs (1), (2),

$$
\begin{gathered}
\sigma=h \rho N_{A} / M_{n} \\
D=(4 / \pi \sigma)^{1 / 2}
\end{gathered}
$$

where $\rho$ is the density of polymer $(1.4136 \mathrm{~g} / \mathrm{mL})^{20}$ and $N_{A}$ is Avogadro's number. The expected polymer chain conformation in good solvent was deduced from the comparison of $D$ with the corresponding gyration radius $\left(R_{g}\right)$ of the unperturbed chains calculated from eq (3),

$$
R_{g}=b .(D P / 6)^{1 / 2}
$$


where $D P$ is the degree of polymerization and $b$ is the segment length $(0.6 \mathrm{~nm})$. The grafting parameters were summarized in Table 2. The ellipsometric thickness of non-cross-linked PIL layers (Ba-c) were measured as $11 \pm 2,14 \pm 4$, and $18 \pm 3 \mathrm{~nm}$, while their grafting densities were calculated as $0.67,0.72$, and 0.80 chain $/ \mathrm{nm}^{2}$, respectively. A comparison of the average distance between the grafting points with twice the radius of gyration of the PIL chain resulted in a ratio of 0.32 (less than 1.0), which means that the grafted chains are indeed in a stretched and brush-like conformation. As a result of static water contact angle measurements, it was determined that all surfaces show a similar hydrophilic character, and the contact angle is about $45^{\circ}$.

Table 2. Grafting parameters of PIL layers

\begin{tabular}{|c|c|c|c|c|c|c|c|c|c|}
\hline & $\begin{array}{c}\text { Conversion } \\
(\%)^{\mathrm{a}}\end{array}$ & $\begin{array}{c}M_{n, \text { theo }} \times 10^{4} \\
\left(\text { g.mol }^{-1}\right)^{\mathbf{b}}\end{array}$ & $\begin{array}{c}M_{n, S E C} \times 10^{4} \\
(\text { g.mol } \\
\end{array}$ & $M_{w} / M_{n}$ & DP & $\begin{array}{c}h \\
(\mathbf{n m})^{\mathrm{c}}\end{array}$ & $\begin{array}{c}\sigma \\
(\text { chains.nm } \\
-2\end{array}$ & $\begin{array}{c}D \\
(\mathbf{n m})\end{array}$ & $\begin{array}{c}\boldsymbol{R}_{g} \\
(\mathbf{n m})\end{array}$ \\
\hline $\mathbf{B a}$ & 63 & 1.48 & 1.40 & 1.28 & 60 & $11 \pm 2$ & 0.67 & 1.38 & 1.89 \\
\hline Bb & 74 & 1.74 & 1.65 & 1.25 & 71 & $14 \pm 4$ & 0.72 & 1.33 & 2.06 \\
\hline Bc & 82 & 1.95 & 1.80 & 1.20 & 82 & $18 \pm 3$ & 0.80 & 1.26 & 2.22 \\
\hline
\end{tabular}

${ }^{a}$ The conversion of the polymerization was determined gravimetrically.

${ }^{\mathrm{b}} M_{n \text {,theo }}=\left(\right.$ conversion $\left.x c_{\text {monomer }} \times M_{\text {monomer }} / c_{R A F T \text { agent }}\right)+M_{R A F T \text { agent }}$,

Monomer/RAFT agent/Initiator: 200:2:1.

${ }^{\mathrm{c}}$ Dry thickness of PIL layers.

\subsection{Swelling and Durability of PILs layer}

To determine the swelling ratio in deionized water of Ba-c and CBa-c layers, the dry and wet thicknesses of the surfaces were measured by ellipsometry. The thickness swelling ratio, $\alpha$, is defined:

$$
\alpha=h_{s} / h_{d}
$$

where $h_{s}$ is the thickness of the swollen brush, and $h_{d}$ is the thickness of the dry brush. Figure 3 shows the change in surfaces' swelling ratio as a function of grafting density and cross-linker ratio. The swelling behavior of cross-linked polymer brushes was observed that the swelling ratio decreased with the increase of the cross-linker ratio in accordance with the literature. ${ }^{20,29}$

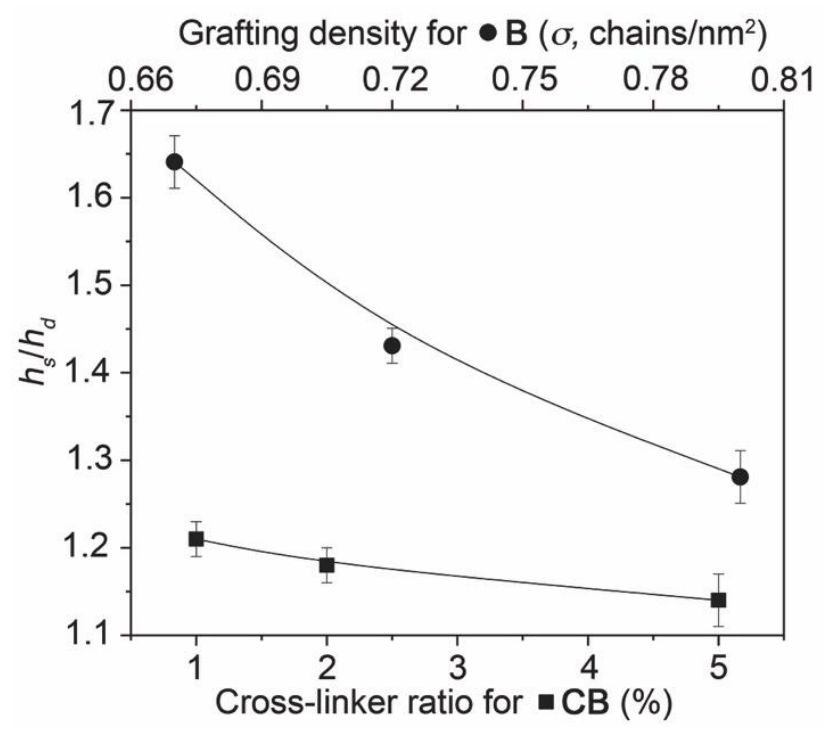

Figure 3. Swelling ratio $\left(h_{s} / h_{d}\right)$ of Ba-c and CBa-c layers in deionized water

One of the most important factors directly affecting the area of use of prepared surfaces is stability. For this reason, static contact angle measurements and hysteresis values were used in addition to ellipsometric thicknesses to determine the prepared surfaces' stability. After the surfaces were 
prepared, their ellipsometric thickness, water contact angles, and hysteresis values were determined, and the results for each surface were presented in the previous section. Prepared and characterized surfaces were incubated in a PBS buffer at $37^{\circ} \mathrm{C}$ for 72 hours in a homemade rotary flow system. The surfaces were then dried, and ellipsometric thicknesses, water contact angles, and hysteresis values were determined.

The ellipsometric thicknesses of polymer brushes and brush gels before and after incubation are given in Table 3. After incubation, the polymer brushes' (Ba-c) thickness was $7 \pm 3,8 \pm 5$, and $11 \pm 4 \mathrm{~nm}$, respectively. After incubation, the ellipsometric thickness of polymer brushes reduced by about $40 \%$, while this was only $15 \%$ for cross-linked polymer brushes. The ellipsometric thicknesses of cross-linked polymer brushes (CBa-c) after incubation were measured at $11 \pm 5,14 \pm 3$, and $18 \pm 4 \mathrm{~nm}$. However, the loss of ellipsometric thickness decreased from $21 \%$ to $14 \%$ with increasing the cross-linking ratio. This indicates that the stability of brush gels was increased with the increase of the cross-linker ratio.

Table 3. The ellipsometric thickness, water contact angle, and hysteresis of Ba-c and CBa-c layers before and after incubation

\begin{tabular}{|c|c|c|c|c|c|c|}
\hline & \multicolumn{2}{|c|}{ Thickness (nm) ${ }^{\mathrm{a}}$} & \multicolumn{2}{|c|}{ Contact Angle $\left(^{\circ}\right)$} & \multicolumn{2}{|c|}{ Hysteresis $\left({ }^{\circ}\right)^{b}$} \\
\hline & Before & After & Before & After & Before & After \\
\hline $\mathbf{B a}$ & $11 \pm 2$ & $7 \pm 3$ & $45 \pm 6$ & $43 \pm 7$ & $14 \pm 4$ & $21 \pm 5$ \\
\hline Bb & $14 \pm 4$ & $8 \pm 5$ & $46 \pm 5$ & $44 \pm 7$ & $12 \pm 5$ & $23 \pm 4$ \\
\hline Bc & $18 \pm 3$ & $11 \pm 4$ & $44 \pm 7$ & $42 \pm 6$ & $17 \pm 5$ & $29 \pm 7$ \\
\hline $\mathbf{C B a}$ & $14 \pm 3$ & $11 \pm 5$ & $48 \pm 4$ & $47 \pm 5$ & $18 \pm 4$ & $19 \pm 6$ \\
\hline CBb & $17 \pm 5$ & $14 \pm 3$ & $45 \pm 6$ & $45 \pm 4$ & $15 \pm 4$ & $17 \pm 5$ \\
\hline CBc & $21 \pm 4$ & $18 \pm 4$ & $44 \pm 6$ & $43 \pm 6$ & $11 \pm 3$ & $12 \pm 4$ \\
\hline
\end{tabular}

${ }^{\mathrm{a}}$ Dry thickness of PIL layers.

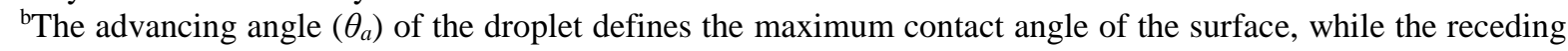
angle $\left(\theta_{r}\right)$ defines the minimum contact angle of the surface (where hysteresis is defined by $\Delta \theta=\theta_{a}-\theta_{r}$ ).

The water contact angles and hysteresis values of surfaces were also studied before and after incubation. The hysteresis values increased due to increasing surface roughness. It is known that the hydrophobic and hydrophilic character of the surfaces increases with surface roughness. The separated chains from the surface caused a decrease in ellipsometric thickness while increasing surface roughness and hysteresis values. For cross-linked polymer brushes, the hysteresis values decreased from $19 \pm 6^{\circ}$ to $12 \pm 4^{\circ}$ with an increased cross-linker ratio. The ellipsometric thickness and hysteresis values showed that the cross-linked polymer brush surface morphology did not change significantly.

\section{Conclusion}

In this study, PILs brushes and brush gels were prepared by RAFT polymerization. The chemical compositions of the prepared surfaces were determined by XPS. The grafting parameters of PIL brushes (Ba-c) were calculated using ellipsometric thickness and molecular weight of the polymers. It was determined that non-crosslinked polymer brushes were in brush conformation. The brush conformation of Ba-c was also confirmed with AFM images. The surface morphologies of cross-linked polymer brushes (CBa-c) were close to polymer brushes, but the RMS values were higher. The observed results demonstrate that the swelling ratio of PIL layers decreased with increasing grafting density and crosslinker ratio. The cross-linked polymer brushes showed that they could gather the features of polymer brushes and gels.

\section{Acknowledgements}

This work was supported in part by The Scientific and Technological Research Council of Turkey-TUBITAK (Project No. 219Z270). 


\section{ORCID}

Selin Kinali-Demirci: 0000-0001-5363-9412

Serkan Demirci: $\underline{\text { 0000-0002-4753-2069 }}$

\section{References}

[1] Plechkova, N. V.; Seddon, K. R. Applications of ionic liquids in chemical industry. Chem. Soc. Rev. 2008, 37, 123-150.

[2] Zhang, Q.; Zhang, S.; Deng, Y. Recent advances in ionic liquid catalysis. Green Chem. 2011, 13, 26192637.

[3] Watanabe, M.; Thomas, M. L.; Zhang, S.; Ueno, K.; Yasuda, T.; Dokko K. Application of ionic liquids to energy storage and conversion materials and devices. Chem. Rev. 2017, 117, 7190-7239.

[4] Zhu, S.; Wu, Y.; Chen, Q.; Yu, Z.; Wang, C.; Jin, S.; Ding, Y.; Wu, G. Dissolution of cellulose with ionic liquids and its application: A mini-review. Green Chem. 2006, 8, 325-327.

[5] Devasurendra, A. M.; Zhang, C.; Young, J. A.; Tillekeratne, L. M. V.; Anderson, J. L.; Kirchhoff, J. R. Electropolymerized pyrrole-based conductive polymeric ionic liquids and their application for solid-phase microextraction. ACS Appl. Mater. Interfaces 2017, 9, 24955-24963.

[6] Grygiel, K.; Lee, J.-S.; Sakaushi, K.; Antonietti, M.; Yuan, Y. Thiazolium poly(ionic liquid)s: Synthesis and application as binder for lithium-ion batteries. ACS Macro Lett. 2015, 4, 1312-1316.

[7] Dong, Y.-S.; Xiong, X.-H.; Lu, X.-W.; Wu, Z.-Q.; Chen, H. Antibacterial surfaces based on poly(cationic liquid) brushes: Switchability between killing and releasing via anion counterion switching. J. Mater. Chem. B 2016, 4, 6111-6116.

[8] Tauber, K.; Dani, A.; Yuan, J. Covalent cross-linking of porous poly(ionic liquid) membrane via a triazine network. ACS Macro Lett. 2017, 6, 1-5.

[9] Giussi, J. M.; Cortez, M. L.; Marmisolle, W. A.; Azzaroni, O. Practical use of polymer brushes in sustainable energy applications: interfacial nanoarchitectonics for high-efficiency devices. Chem. Soc. Rev. 2019, 48, 814-849.

[10] Demirci, S.; Kinali-Demirci, S.; Jiang, S. A switchable polymer brush system for antifouling and controlled detection. Chem. Commun. 2017, 53, 3713-3716.

[11] Wang, P.; Koberstein, J. T., Morphology of immiscible polymer blend thin films prepared by spin-coating. Macromolecules 2004, 37, 5671-5681.

[12] Peng, S.; Bhushan, B. Smart polymer brushes and their emerging applications. RSC Adv. 2012, 2, 85578578.

[13] Demirci, S.; Caykara, T. High density cationic polymer brushes from combined "click chemistry" and RAFT-mediated polymerization. J. Polym. Sci., Part A: Polym. Chem. 2012, 50, 2999-3007.

[14] Chen, W.-L.; Cordero, R.; Tran, H.; Ober, C. K. 50th Anniversary Perspective: Polymer Brushes: Novel Surfaces for Future Materials. Macromolecules 2017, 50, 4089-4113.

[15] Ozturk, E. A.; Eroglu, M. S.; Caykara, T. Synthesis of hyaluronated poly(exo-7-oxabicyclo[2.2.1] hept-5-en-2,3-dicarboxylic anhydride) brushes via a combination of surface-initiated ring-opening metathesis polymerization and thiol-ene click reaction. Chemical Papers 2020. DOI: $10.1007 / \mathrm{s} 11696-020-01418-5$

[16] Demirci, S.; Kinali-Demirci, S.; Caykara, T. Stimuli-responsive diblock copolymer brushes via combination of "click chemistry" and living radical polymerization. J. Polym. Sci., Part A: Polym. Chem. 2013, 51, 2677-2685.

[17] Edmondson, S.; Osborne, V. L.; Huck, W. T. S. Polymer brushes via surface-initiated polymerizations. Chem. Soc. Rev. 2004, 33, 14-22.

[18] Zhao, B.; Brittain, W. J. Polymer brushes: surface-immobilized macromolecules. Prog. Polym. Sci. 2000, $25,677-710$.

[19] Welch, M. E.; Ober, C. K. Responsive and patterned polymer brushes. J. Polym. Sci., Part A: Polym. Chem. 2013, 51, 1457-1472.

[20] Demirci, S.; Kinali-Demirci, S.; VanVeller, B. Surface-grafted polymeric ionic liquids with tunable morphology via in/ex situ cross-linking methods. ACS Macro Lett. 2020, 9, 1806-1811.

[21] Dehghani, E. S.; Spencer, N. D.; Ramakrishna, S. N.; Benetti, E. M. Cross-linking polymer brushes with ethylene glycol-containing segments: influence on physicochemical and antifouling properties. Langmuir 2016, 32, 10317-10327.

[22] Demirci, S. Crosslinked-polymer brushes with switchable capture and release capabilities. Polymers 2018, $10,956$.

[23] Moncy, P.; Klok, H.-A. Reversibly cross-linking polymer brushes using interchain disulfide bonds. Macromolecules 2020, 53, 731-740. 
Surface-grafted polymer brushes and brush-gels

[24] Zhang, J.; Xu, D.; Guo, J.; Sun, Z.; Qian, W.; Zhang, Y.; Yan, F. CO2 responsive imidazolium-type poly(ionic liquid) gels. Macromol. Rapid Commun. 2016, 37, 1194-1199.

[25] Taghavikish, M.; Subianto, S.; Dutta, N. K.; de Campo, L.; Mata, J. P.; Rehm, C.; Choudhury, N. R. Polymeric ionic liquid nanoparticle emulsions as a corrosion inhibitor in anticorrosion coatings. ACS Omega 2016, 1, 29-40.

[26] Demirci, S.; Kinali-Demirci, S.; Caykara, T. Novel pH-responsive mixed-charge copolymer brushes based on carboxylic acid and quaternary amine monomers. J. Polym. Sci., Part A: Polym. Chem. 2013, 51, 16121619.

[27] Demirci, S.; Caykara, T. RAFT-mediated synthesis of cationic poly[(ar-vinylbenzyl)trimethylammonium chloride] brushes for quantitative DNA immobilization. Mater. Sci. Eng., C 2013, 33, 111-120.

[28] Demirci, S.; Kinali-Demirci, S.; VanVeller, B. Controlled supramolecular complexation of cyclodextrinfunctionalized polymeric ionic liquid brushes. ACS Appl. Polym. Mater. 2020, 2, 751-757.

[29] Malham, I. B.; Bureau, L. Density effect on collapse, compression and adhesion of thermoresponsive polymer brushes. Langmuir 2010, 26, 4762-4768.

\section{A C G \\ publications \\ (C) 2021 ACG Publications}

